\title{
Robustness Evaluation on Earthquake Response of Base-Isolated Buildings with Uncertain Structural Properties under Long-Period Ground Motions
}

\author{
Kohei Fujita ${ }^{1}$, and Izuru Takewaki ${ }^{2 *}$ \\ ${ }^{1}$ Postdoctoral Fellow, Kyoto University, Kyotodaigaku-Katsura, Nishikyo-ku, Kyoto 615-8540 Japan. \\ ${ }^{2}$ Professor of Department of Architecture and Architectural Engineering, Kyoto University, Kyotodaigaku-Katsura, \\ Nishikyo-ku, Kyoto 615-8540 Japan, *Corresponding author.
}

doi:10.5618/arch.2012.v1.n1.5 || Received: 20-03-2012, Accepted: 29-03-2012, Available online: 30-03-2012

\begin{abstract}
Robustness of a base-isolated building with uncertain structural parameters under long-period ground motions is investigated by uncertainty analysis methodologies. It is shown that the critical combination of structural parameters maximizing the objective function can be derived by a proposed uncertainty analysis method, i.e., combining the so-called URP (Updated Reference-Point) method with a response surface method. The practical application of the proposed robustness evaluation methodology is presented through an example of a base-isolated building model with the natural rubber bearing (NRB) and lead rubber bearing (LRB) isolators. The results from the proposed methods are compared with those from the Monte Carlo simulation under various long-period ground motions. It is shown that the proposed URP method combined with the response-surface type can provide a reliable and accurate evaluation of robustness for the earthquake response bound without hard computational load.
\end{abstract}

$\begin{array}{ll}\text { Abbreviations: } \\ \text { LRB } & \text { lead rubber bearing } \\ \text { MCS } & \text { Monte Carlo simulation } \\ \text { NRB } & \text { natural rubber bearing } \\ \text { RSM } & \text { response surface method } \\ \text { URP } & \text { Updated Reference-Point }\end{array}$

Keywords: Interval analysis, Uncertainty, Base-isolation, Earthquake response, Robustness, Long-period ground motion

\section{Introduction}

An efficient methodology is proposed to evaluate the robustness (variability of response) of a base-isolated building with uncertain structural properties under long-period ground motions (see Fig.1). It is well known that base-isolated buildings have large structural uncertainties due to wide variability of structural properties caused by temperature and frequency dependencies, manufacturing errors and aging effect [1-3]. Furthermore, after the worst disaster of the 2011 off the Pacific coast of Tohoku earthquake in Japan, it is under discussion that base-isolated buildings are vulnerable against unexpected long-period ground motions.

Under these circumstances, it is desired to evaluate the response variability caused by those structural variability and uncertain ground motions [4-6]. The method based on the convex model may be one possibility [7]. Independently, Kanno and Takewaki [8, 9] proposed an efficient and reliable method for evaluating the robustness of structures under uncertainties based on the concept of the robustness function [10, 11]. However it does not appear that an efficient and reliable method for evaluating the robustness of structures has been proposed.

An interval analysis is believed to be one of the most efficient and reliable method to respond to this requirement. While a basic assumption of "inclusion monotonic" is introduced in usual interval analysis, a possibility should be taken into account of occurrence of the extreme value of the objective function in an inner feasible domain of the interval parameters for more accurate and reliable evaluation of the objective function. It is shown that the critical combination of the structural parameters can be derived explicitly by maximizing the approximate objective function and using the proposed method combining the URP (Updated Reference-Point) method $[12,13]$ with a response surface method.

In order to investigate the validity of the proposed 
robustness evaluation methodologies, numerical analyses are conducted for 20-story base-isolated building models with natural rubber bearing isolators, lead rubber bearing isolators and passive dampers for various long-period ground motions recorded in recent years. The maximum displacement in the base-isolation story and the maximum acceleration at the building top floor are chosen as the objective function. The Monte Carlo simulation (MCS) shows that, since elastic-plastic behaviors are taken into account, the URP method combined with a response surface method is more accurate and reliable for the evaluation of response bounds without hard computational effort.
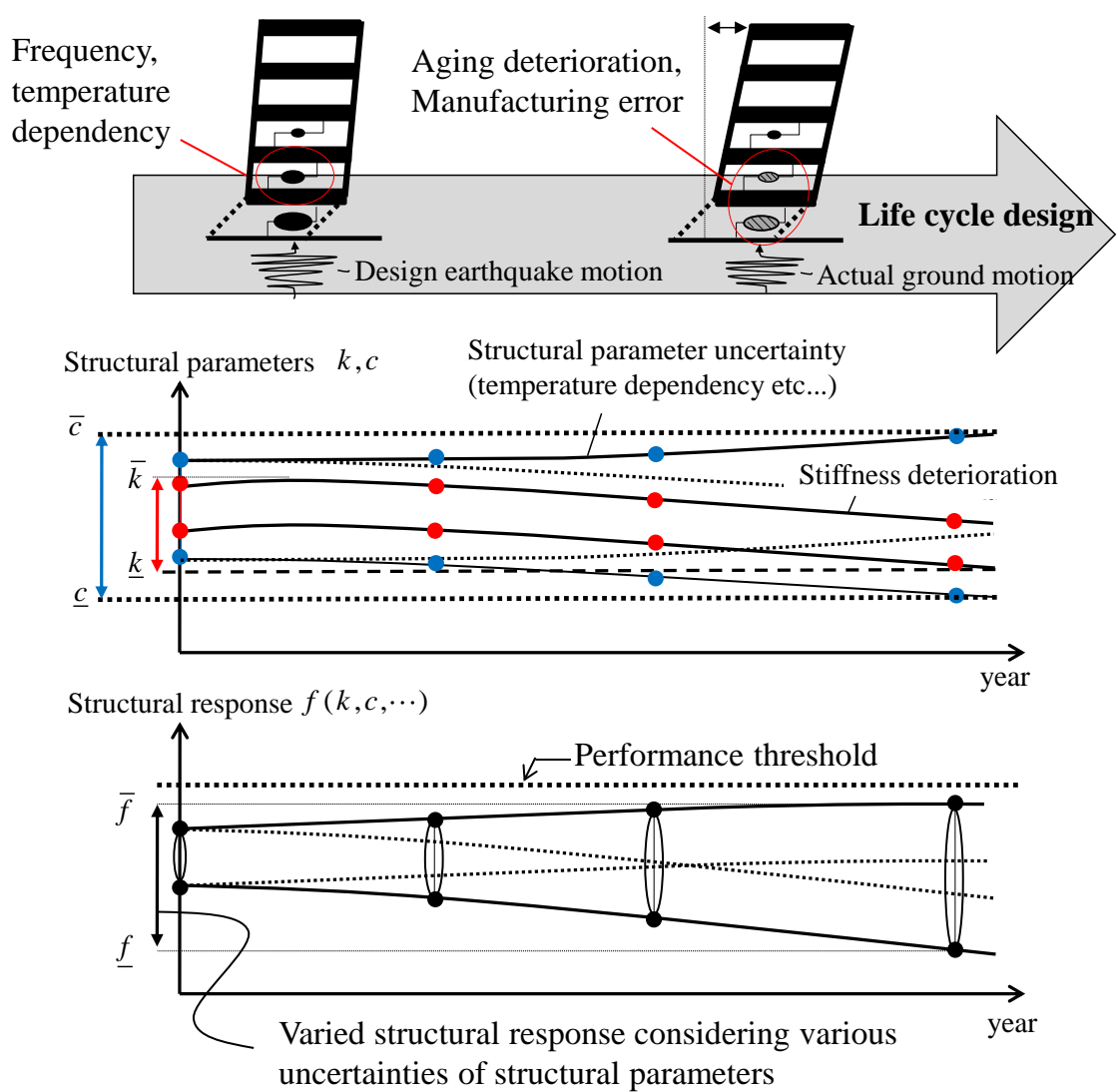

Fig.1 Variation of structural response under uncertain structural parameters in base-isolated building

\section{Interval analysis of uncertain structural parameters.}

For evaluating the robustness of structures with uncertain parameters under uncertain disturbances, various uncertainty analysis methods have been proposed so far (for example, [5, 10, 11, 14-17]). An interval analysis may be one of the most effective methods for the analysis under uncertainty. The interval analysis is aimed at finding the upper and lower bounds of the objective function for given intervals of uncertain parameters.

Let $\mathbf{X}$ denote a set of uncertain parameters. The components of this set are referred to as interval variables and expressed as

$$
\mathbf{X}^{I}=\left\{\left[X_{i}^{c}-\Delta \underline{X}_{i}, X_{i}^{c}+\Delta \bar{X}_{i}\right]\right\} \quad\left(i=1, \cdots, N_{x}\right)
$$

where ( $)^{I}$ denotes an interval variable and $[a, b]$ indicates that $a$ and $b$ are the lower and upper bounds of the interval parameter. In Eq.(1), ( $)^{c}, \Delta \bar{X}, \Delta \underline{X}$ and $N_{x}$ denote the nominal value, the upper-side band width, the lower-side band width and the number of interval variables, respectively.

The following representation of the objective function in terms of interval variables is called the interval extension. 


$$
f^{I}(\mathbf{X})=f\left(\mathbf{X}^{I}\right)=f\left(\left[X_{1}^{c}-\Delta \underline{X}_{1}, X_{1}^{c}+\Delta \bar{X}_{1}\right], \cdots,\left[X_{N_{x}}^{c}-\Delta \underline{X}_{N_{x}}, X_{N_{x}}^{c}+\Delta \bar{X}_{N_{x}}\right]\right)
$$

In Eq.(2), the upper and lower bounds of the objective function are assumed to be given by the combination of end points of interval variables. In usual interval analysis, a basic assumption of "inclusion monotonic" is introduced and the following relation with respect to the objective function $f$ is assumed

$$
\left\{f(\mathbf{X}): X_{i} \in X_{i}^{I}, i=1,2, \cdots, N_{x}\right\} \subseteq f\left(X_{1}^{I}, X_{2}^{I}, \cdots, X_{N_{X}}^{I}\right)
$$

Since the right-hand side of Eq.(3) corresponds to Eq.(2), the maximum and minimum values of the objective function occur at the end points of interval variables. Fig.2(a) presents a monotonic contour map of the objective function for two design variables $X_{1}$, $X_{2}$. In this case, an exact solution is included in the combination of end points of interval parameters. Therefore an exact solution can be obtained by conducting the response analysis for all the combinations of end points $\bar{X}_{i}=X_{i}^{c}+\Delta \bar{X}_{i}$ and $\underline{X}_{i}=X_{i}^{c}-\Delta \underline{X}_{i}$ of interval parameters. The computational load (number of repetition) is given by $2^{N_{x}}$. However this repetition becomes huge for a large number of interval parameters.

In order to overcome this difficulty, some approaches have been proposed, e.g., a method of approximating the inverse of the stiffness matrix by Neumann expansion ([15]), or a method of approximating the objective function by converged series expansion ([16]). Furthermore Chen et al. [17] proposed a method of using a first-order Taylor series expansion and a second-order Taylor series expansion with diagonal components only in approximating the objective function.

On the other hand, when the basic assumption "inclusion monotonic" cannot be applied to the objective function, it is not appropriate to evaluate the objective function only at the end points of interval parameters. Fig.2(b) shows the two-variable examples of non-monotonic contour map of the objective function. In this case, a sequential quadratic programming method or a response surface method has to be introduced in transforming the original problem of finding the upper and lower bounds of the objective function into an optimization problem. This procedure causes a large amount of computational work.

\section{Interval analysis using Taylor series expansion}

As stated before, it is often the case that the objective function becomes non-monotonic ones with respect to interval parameters. A general problem of interval analysis may be stated as follows (equation 4). (a)

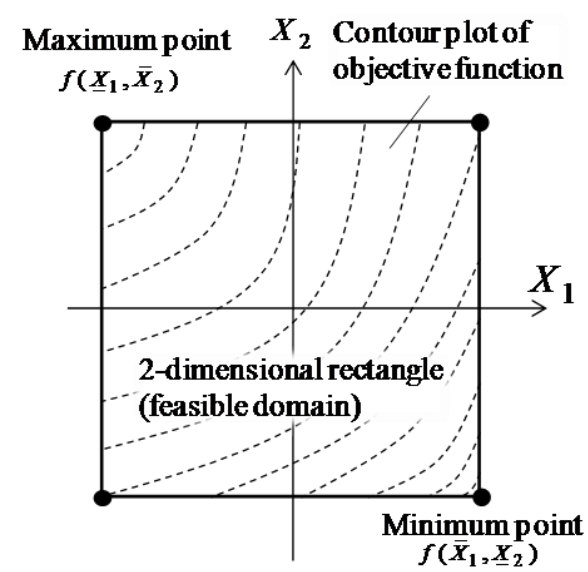

(b)

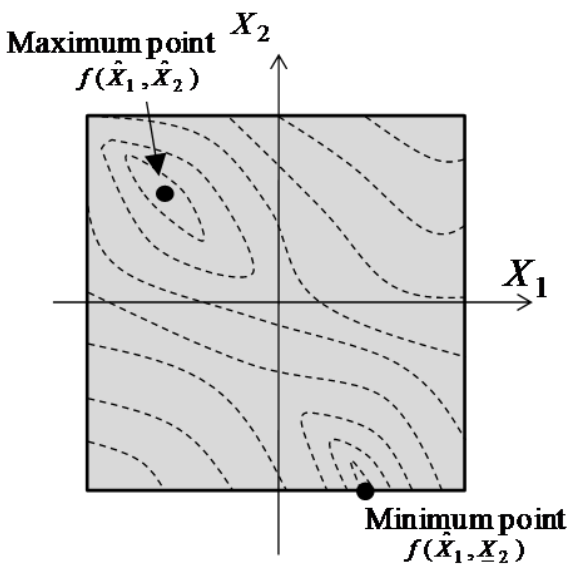

Fig.2 Comparison of the properties of the objective function under uncertain design parameters (two-dimension),

(a) Inclusion monotonic, (b) non-monotonic 
Find $\mathbf{X}$

so as to maximize or minimize $f(\mathbf{X})$

$$
\text { subject to } \mathbf{X} \in \mathbf{X}^{I}
$$

Eq.(4) indicates a constrained optimization problem and various methods can be used to solve this as stated before. However there are many difficulties in the computational time or convergence.

In this paper, a new method as a framework of the interval analysis is proposed which can overcome the difficulty in problems with non-monotonic properties of objective functions. For preparing for this, interval analysis using first and second-order Taylor series expansion is explained in Section 3.1 and 3.2.

3.1 Interval analysis using first-order Taylor series expansion. The upper and lower bounds of the objective function by the first-order Taylor series expansion can be obtained as equation (5), where the ( ), ${ }_{x i}$ denote the differentiation of the objective function at the nominal value,

$$
\partial f(\mathbf{X}) /\left.\partial X_{i}\right|_{X_{i}=X_{i}^{c}}
$$

i.e. the first-order sensitivity of the objective function with respect to an interval parameter.

$$
\bar{f}=f\left(\mathbf{X}^{c}\right)+\sum_{i=1}^{N_{x}}\left|f_{, X_{i}} \Delta X_{i}\right|, \quad \underline{f}=f\left(\mathbf{X}^{c}\right)-\sum_{i=1}^{N_{x}}\left|f_{, X_{i}} \Delta X_{i}\right|
$$

3.2 Interval analysis using second-order Taylor series expansion. An approximation of the objective function around the nominal value can be expressed as the second-order Taylor series expansion as equation (6), where ( ), $X i X j$ denotes the second-order differentiation of the objective function at the nominal value. Following Chen et al. (2009), let us approximate the objective function around the nominal value by the second-order Taylor series expansion with diagonal terms only.

In Eq.(7), each increment of the objective function can be derived independently by each interval parameter.
For example, the increment with respect to $X_{1}$ may be expressed by equation (8).

In order to obtain the upper and lower bounds of the function of Eq.(7), it is necessary to evaluate the maximum and minimum values of $\Delta f_{i} \quad\left(i=1, \cdots, N_{x}\right)$ and sum up those values. For example, the upper and lower bounds of $\Delta f_{1}$ can be obtained as follows by doing the interval extension of Eq.(8).

From Eq.(9), the computation of $\Delta f_{1}$ for the upper and lower bounds $\bar{X}_{1}, \underline{X}_{1}$ of $X_{1}$ leads to the upper and lower bounds of $\Delta f_{1}$.

$$
\begin{gathered}
f^{*}(\mathbf{X})=f\left(\mathbf{X}^{c}\right)+\sum_{i=1}^{N_{x}} f_{, X_{i}}\left(X_{i}-X_{i}^{c}\right)+\frac{1}{2} \sum_{i=1}^{N_{x}} \sum_{j=1}^{N_{x}} f_{, X_{i} X_{j}}\left(X_{i}-X_{i}^{c}\right)\left(X_{j}-X_{j}^{c}\right) \\
f^{* *}(\mathbf{X})=f\left(\mathbf{X}^{c}\right)+\sum_{i=1}^{N_{x}}\left\{f_{, X_{i}}\left(X_{i}-X_{i}^{c}\right)+\frac{1}{2} f_{, X_{i} X_{i}}\left(X_{i}-X_{i}^{c}\right)^{2}\right\} \\
\Delta f_{1}\left(X_{1}, X_{2}^{c}, \cdots, X_{N_{x}}^{c}\right)=f_{, X_{1}}\left(X_{1}-X_{1}^{c}\right)+\frac{1}{2} f_{, X_{1} X_{1}}\left(X_{1}-X_{1}^{c}\right)^{2} \\
\Delta f_{1}^{I}\left(X_{1}^{I}, X_{2}^{c}, \cdots, X_{N_{x}}^{c}\right)=\left[\begin{array}{l}
\min \left[\Delta f_{1}\left(\bar{X}_{1}, X_{2}^{c}, \cdots, X_{N_{x}}^{c}\right), \Delta f_{1}\left(\underline{X}_{1}, X_{2}^{c}, \cdots, X_{N_{x}}^{c}\right)\right], \\
\left.\max \left[\Delta f_{1}\left(\bar{X}_{1}, X_{2}^{c}, \cdots, X_{N_{x}}^{c}\right), \Delta f_{1}\left(\underline{X}_{1}, X_{2}^{c}, \cdots, X_{N_{x}}^{c}\right)\right]\right]
\end{array}\right.
\end{gathered}
$$


3.3 Interval analysis considering non-monotonic property of objective function. In the method explained in previous sections, it was assumed that the maximum and minimum values of the objective function can be obtained at the end points of interval parameters. However, when the level of uncertainty is large, the approximation by Taylor series expansion may lead to large errors. In this section, two different methods to overcome this difficulty will be shown.

\subsubsection{Estimation of the variation of the objective} function by 2nd-order Taylor series expansion. If the critical combination of interval parameters maximizing the objective function can be predicted, it becomes possible to evaluate the robustness accurately. Therefore, the Taylor series approximation with diagonal elements only is used to predict this critical combination. As for the variation $\Delta f_{i}\left(d X_{i}\right)$ for $X_{i}$, let us regard $d X_{i}=X_{i}-X_{i}^{c}$ as a variable satisfying $-\Delta \underline{X}_{i} \leq d X_{i} \leq \Delta \bar{X}_{i}$. Then the variation $\Delta f_{i}\left(d X_{i}\right)$ can be rewritten as equation (10).

The function of Eq.(10) is a quadratic function and the value $d X_{i}$ maximizing or minimizing $\Delta f$ can be obtained explicitly. Consider the case $f_{X i} X i<0$. If the notation $\Delta \bar{X}_{i}=\Delta \underline{X}_{i}=\Delta X_{i}$ is used, the interval parameter maximizing $\Delta f_{i}$ can be obtained as equation (11).

The evaluation of sensitivities $f_{, X i}$ and $f_{, X i}$ i is made at the nominal model (reference point). It should be noted that the correlation among interval parameters is not taken into account. In this paper, a method called the URP (updated reference-point) method [12, 13] is used. This method updates the reference point step by step. The flowchart for finding the upper bound of the objective function is shown in Fig.3 (a).

$$
\begin{gathered}
\Delta f_{i}\left(d X_{i}\right)=\frac{1}{2} f_{, X_{i} X_{i}}\left(d X_{i}+f_{, X_{i}} / f_{, X_{i} X_{i}}\right)^{2}-f_{, X_{i}}{ }^{2} / 2 f_{, X_{i} X_{i}} \\
\hat{X}_{i}= \begin{cases}X_{i}^{c}-f_{, X_{i}} / f_{, X_{i} X_{i}}\left(\left|f_{, X_{i}} / f_{, X_{i} X_{i}}\right| \leq \Delta X_{i}\right) \\
X_{i}^{c}+\Delta X_{i} & \left(-f_{, X_{i}} / f_{, X_{i} X_{i}} \geq 0,\left|f_{, X_{i}} / f_{, X_{i} X_{i}}\right|>\Delta X_{i}\right) \\
X_{i}^{c}-\Delta X_{i} & \left(-f_{, X_{i}} / f_{, X_{i} X_{i}}<0,\left|f_{, X_{i}} / f_{, X_{i} X_{i}}\right|>\Delta X_{i}\right)\end{cases}
\end{gathered}
$$

3.3.2 Estimation of the variation of the objective function by response surface approach. In the proposed method using Taylor series expansion as explained in previous sections, the accuracy of the robustness evaluation depends directly on the reliability of the numerical sensitivity analysis. For this reason, when the evaluation of numerical sensitivities has some difficulties due to the elastic-plastic structural property of isolators, another URP method should be introduced where the variation of the objective function is estimated using a response surface method (RSM). RSM is well known as a statistical response evaluation technique which can provide the continuous variation of the objective function by several response samplings. In this paper, three response values $\bar{f}$ evaluated at the nominal, lower and upper ends of an interval parameter $X_{i}$ are used and the following 2nd-order polynomial model is introduced.

$$
f\left(X_{i}\right)=a_{1}+a_{2} X_{i}+a_{3} X_{i}^{2}
$$

In Eq.(12) $a_{i}(i=1,2,3)$ are unknown variables derived from reference responses. By using this approximation of the objective function, we can apply the URP method to the uncertainty analysis as shown in section 3.3.1. Fig.3(b) shows the flowchart of the URP method using RSM.

\section{Application of URP method to base-isolated building model.}

Application of the URP method to the base-isolated building model is shown in this section. The validity of the URP method using 2nd-order Taylor series approximation (Section 3.3.1) and RSM (Section 3.3.2) are demonstrated through the comparison with the other method (Monte Carlo Simulation (MCS)). Although the number of interval parameters as shown later is large in this simulation, the response analyses are conducted for randomly selected combination of end points of interval parameters. 


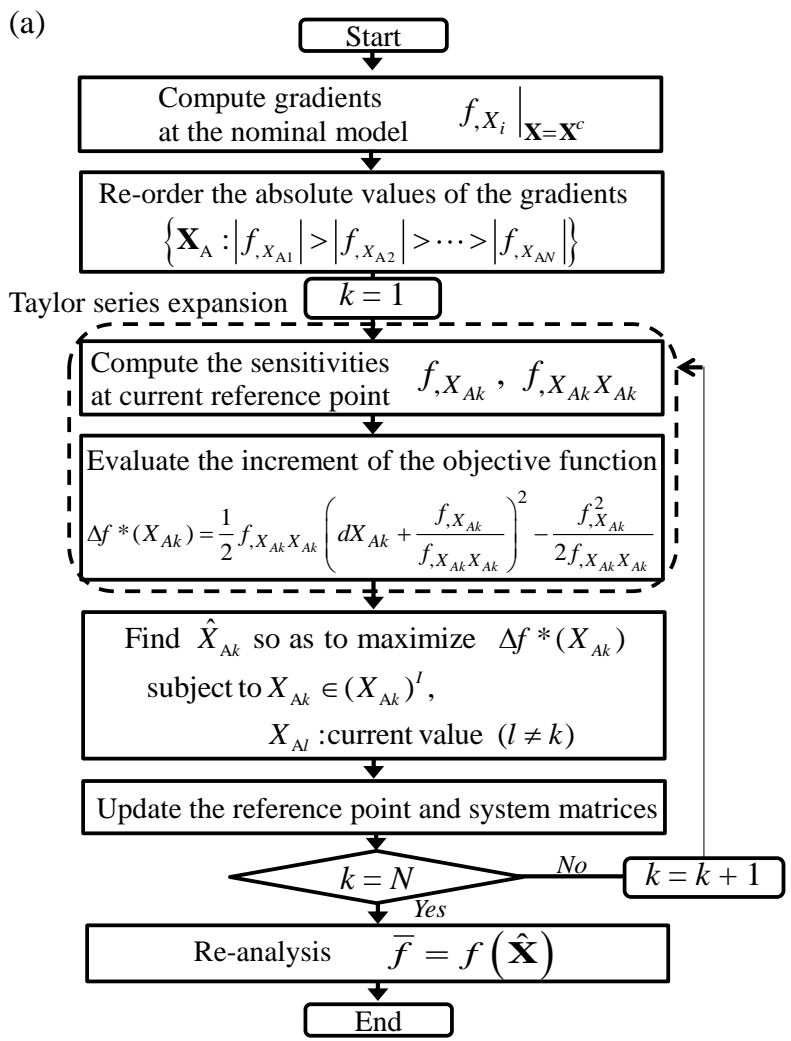

(b)

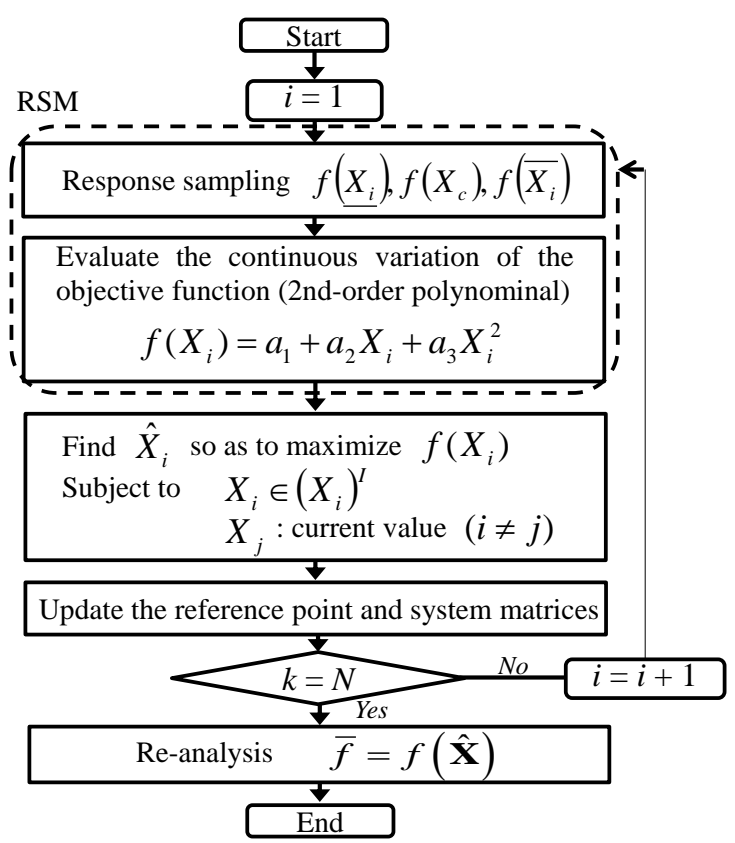

Fig. 3. Flowchart of URP method:

(a) 2nd-order Taylor series approximation; (b) Response surface method (RSM)

4.1 Modeling of base-isolated buildings and uncertainty of structural parameters. Consider an $\mathrm{N}$-story base-isolated shear building model as shown in Fig.4. A simple model is used here to present an essential feature of the robustness evaluation method. Since the use of natural rubber bearings (NRB) only as isolator elements may lead to a higher level of acceleration of top story, both NRB and lead rubber bearings (LRB) are used here. Furthermore, an additional damping in the base-isolation story is to be given by oil dampers.

It is necessary to take into account the response variability (robustness) of the building under earthquake ground motions due to the variability of isolators, dampers and super-structural properties. Table 1 shows an example of variability ratios of isolators of NRB and LRB.

Interval variables in this example are the damping coefficient $c_{0}$ and elastic shear stiffness $k_{0}$ of NRB, the plastic shear stiffness $K_{d}$ and elastic shear stiffness $K_{e}$ of LRB and the damping coefficient vector $\mathbf{c}_{\mathrm{f}}$ and the horizontal stiffness vector $\mathbf{k}_{\mathrm{f}}$ of the super-structure. The set of interval variables is denoted by

$$
\mathbf{X}^{I}=\left\{c_{0}^{I}, k_{0}^{I}, K_{d}^{I}, K_{e}^{I}, \mathbf{c}_{\mathrm{f}}^{I}, \mathbf{k}_{\mathrm{f}}^{I}\right\}
$$

For members with the same properties, it may be appropriate to assign the same variability in each group of members in a building. The present formulation is applicable to such realistic case.

Let us define the ratios of the upper and lower variations of interval variables to the corresponding nominal values.

$$
\begin{aligned}
& \overline{\boldsymbol{\alpha}}=\left\{\frac{\Delta \bar{c}_{0}}{c_{0}^{c}}, \frac{\Delta \bar{k}_{0}}{k_{0}^{c}}, \frac{\Delta \bar{K}_{d}}{K_{d}^{c}}, \frac{\Delta \bar{K}_{e}}{K_{e}^{c}}, \frac{\Delta \bar{c}_{\mathrm{f}_{1}}}{c_{\mathrm{f}_{1}}^{c}}, \cdots, \frac{\Delta \bar{k}_{\mathrm{f}_{1}}}{k_{\mathrm{f}_{1}}^{c}}, \cdots\right\} \\
& \underline{\boldsymbol{\alpha}}=\left\{\frac{\Delta \underline{c}_{0}}{c_{0}^{c}}, \frac{\Delta \underline{k}_{0}}{k_{0}^{c}}, \frac{\Delta \underline{K}_{d}}{K_{d}^{c}}, \frac{\Delta \underline{K}_{e}}{K_{e}^{c}}, \frac{\Delta \underline{c}_{\mathrm{f}_{1}}}{c_{\mathrm{f}_{1}}^{c}}, \cdots, \frac{\Delta \underline{\mathrm{f}}_{1}}{k_{\mathrm{f}_{1}}^{c}}, \cdots\right\}
\end{aligned}
$$


Table 1. Variability ratio of structural properties of isolators (NRB and LRB).

\begin{tabular}{|c|c|c|c|c|c|}
\hline Isolator & $\begin{array}{l}\text { Structural } \\
\text { parameter }\end{array}$ & Bound & $\begin{array}{c}\text { Manufacturing } \\
\text { Error }\end{array}$ & $\begin{array}{l}\text { Temperature } \\
\text { Dependency }\end{array}$ & $\begin{array}{c}\text { Aging } \\
\text { Deterioration }\end{array}$ \\
\hline \multirow{2}{*}{$\begin{array}{c}\text { NRB } \\
\text { (Natural } \\
\text { Rubber Bearing) }\end{array}$} & \multirow{2}{*}{$\begin{array}{c}\text { Elastic shear } \\
\text { modulus }\end{array}$} & Upper & $+10 \%$ & $+6 \%$ & $+10 \%$ \\
\hline & & Lower & $-10 \%$ & $-5 \%$ & $0 \%$ \\
\hline \multirow{4}{*}{$\begin{array}{c}\text { LRB } \\
\text { (Lead Rubber } \\
\text { Bearing) }\end{array}$} & \multirow{2}{*}{$\begin{array}{c}\text { Plastic } \\
\text { Stiffness }\end{array}$} & Upper & $+10 \%$ & $+6 \%$ & $+11 \%$ \\
\hline & & Lower & $-10 \%$ & $-5 \%$ & $0 \%$ \\
\hline & \multirow{2}{*}{$\begin{array}{l}\text { Hysteretic } \\
\text { Strength }\end{array}$} & Upper & $+10 \%$ & $+23 \%$ & $0 \%$ \\
\hline & & Lower & $-10 \%$ & $-21 \%$ & $0 \%$ \\
\hline
\end{tabular}

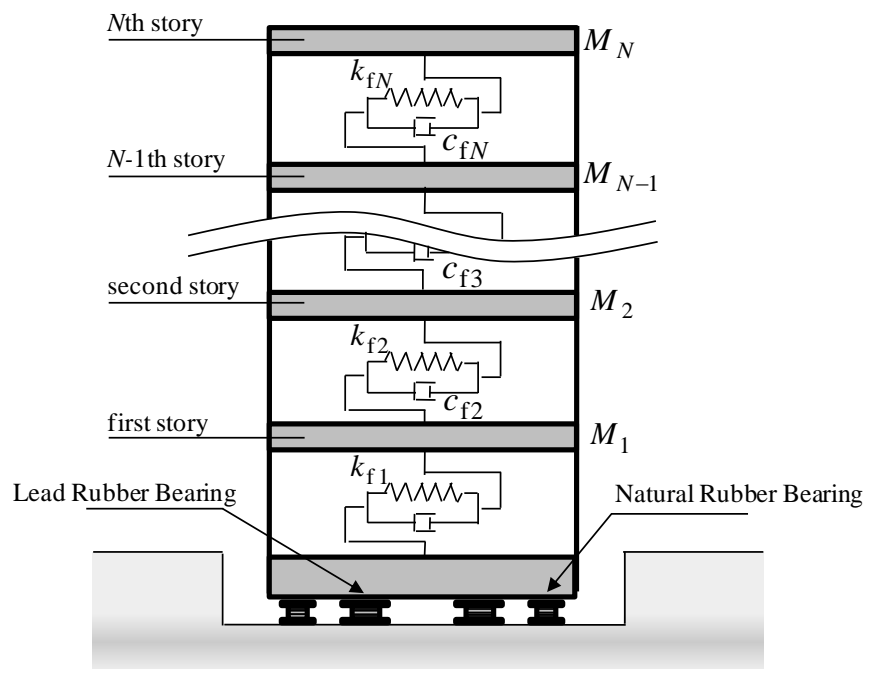

Fig.4 Base-isolated structural model.

\subsection{Property of base-isolated building}

In this section, the structural property of a 20-story base-isolated building $(N=20)$ is summarized. The floor mass per story is $1024 \times 10^{3}(\mathrm{~kg})$. This corresponds to $32 \mathrm{~m} \times 32 \mathrm{~m}$ floor plan $\left(1000 \mathrm{~kg} / \mathrm{m}^{2}\right)$. When the floor mass per story is denoted by $m$ and the lowest-mode of vibration of the super-building with fixed base-isolation story is given by a straight line, then the story stiffness can be expressed as

$$
k_{\mathrm{f}_{i}}=\frac{1}{2}\{N(N+1)-i(i-1)\} m \omega_{1}^{2} \quad(i=1, \cdots, N)(14),
$$

where $\omega_{1}$ is the fundamental natural circular frequency of the super-building with fixed base-isolation story and is given by 3.93rad/s (natural period=1.6s). The super-building is assumed to behave elastically. The damping coefficients of the super-building are given by stiffness-proportional damping with a lowest-mode damping ratio $h=0.02$.
In the selection of the base isolator, the diameter, thickness and shear modulus have to be determined. The required conditions are that 1 ) the stress under dead load is within an allowable value and 2) the deformation capacity and the fundamental natural period are appropriate. When only NRB isolators are used as isolators, the horizontal stiffness $k_{0}$ of the base-isolation story is often determined by specifying the natural circular frequency of the model with the rigid super-building. On the other hand, since LRB isolators have the elastic-plastic characteristic, it is difficult to derive $k_{0}$ for the target natural frequency of the base-isolated building. Table2 shows the property of NRB and LRB isolators of the example model. The natural circular frequency of this base-isolated building is $1.16[\mathrm{rad} / \mathrm{s}]$, i.e. natural period $=5.40 \mathrm{~s}$. The additional damping coefficient of the dampers in the base-isolation story is given by specifying the damping ratio of the model with a rigid super-building as 0.1 . 
Table 2. Parameters of isolators of base-isolated building

\begin{tabular}{|c|c|c|}
\hline & NRB & LRB \\
\hline Diameter of rubber $[\mathrm{mm}]$ & \multicolumn{2}{|c|}{1100} \\
\hline Total rubber thickness $[\mathrm{mm}]$ & 0.390 & 252 \\
\hline Shear modulus of rubber[N/mm $]$ & & 200 \\
\hline Diameter of lead plug[mm $[\mathrm{mm}$ & & 0.588 \\
\hline Shear modulus of lead plug $\left[\mathrm{N} / \mathrm{mm}^{2}\right]$ & & 15 \\
\hline Total number of isolators & 8 & \\
\hline
\end{tabular}

4.3 Input ground motions. Six long-period ground motions are used in this example. These ground motions are scaled to the same maximum ground velocity $=0.5 \mathrm{~m} / \mathrm{s}$. Figs.5 (a)-(f) present those time histories of acceleration and velocity. Fig.6 shows the relationship between the recorded sites and the epicenters of the selected ground motions. In Fig.6, the radius of red or yellow circle represents the moment magnitude. The ground motions depicted by yellow circles represent relatively-large aftershock records (M>6.0) after the 2011 off the Pacific coast of Tohoku earthquake (2011). Furthermore, the velocity response spectra are shown in Fig.7.

4.4 Interval analysis for drift of base-isolation story. The uncertainty analysis is shown here for the maximum drift of base-isolation story. The level of variability in
Eq.(12) is given by equation (15), where $i$ denotes the interval variable number. The case of $i=1$ corresponds to the damping coefficient of oil dampers in the base-isolation story. The case of $i=2$ corresponds to the elastic shear stiffness $k_{0}$ of the NRB. In addition, the case of $i=3$ corresponds to plastic stiffness $K_{d}$ of LRB and the case of $i=4$ corresponds to elastic shear stiffness $K_{e}$ of LRB. Here intervals of $k_{0}$ and $K_{d}$ are treated to be unsymmetrical in increasing and decreasing directions. This is due to the aging effect (hardening) of isolators [2]. Furthermore the case of $i=5, \cdots, N+4$ corresponds to the damping coefficients $\mathbf{c}_{\mathrm{f}}$ of the super-building and the case of $i=N+5, \cdots, 2 N+4$ corresponds to the story stiffness $\mathbf{k}_{\mathrm{f}}$ of the super-building.

$$
\overline{\boldsymbol{\alpha}}=\left\{\begin{array}{ll}
0.3 & (i=1) \\
0.26 & (i=2) \\
0.27 & (i=3) \\
0.26 & (i=4) \\
0.1 & (i=5, \cdots, N+4) \\
0.05 & (i=N+5, \cdots, 2 N+4)
\end{array} \quad \underline{\boldsymbol{\alpha}}= \begin{cases}0.3 & (i=1) \\
0.15 & (i=2) \\
0.15 & (i=3) \\
0.3 & (i=4) \\
0.1 & (i=5, \cdots, N+4) \\
0.05 & (i=N+5, \cdots, 2 N+4)\end{cases}\right.
$$

Fig.8 shows the upper bound of the maximum drift of the base-isolation story compared for various methods (URP methods with 2nd-order Taylor series approximation/with RSM and the Monte Carlo simulation (MCS)). As explained in section 3, the difference between the URP method with 2nd-order Taylor series approximation and that with RSM is how to estimate the variation of the objective function. In the former one, the numerical sensitivities, i.e. the gradient vector and the Hessian matrix, of the objective function are need. On the other hand, in the latter one, a kind of RSM is applied where appropriate response samplings are gathered and the gradient vector and the Hessian matrix are evaluated from the constructed approximate function. In these methods, after deriving an approximate variation of the objective function, the algorithm of the previously proposed uncertainty analysis method, i.e. the URP method [12,13], is applied in the same manner. So as to compare the validity of these proposed methods, MCS has been implemented. In the MCS, the combinations of uncertain parameters are given by randomly selected combination of end points. In Fig.8, both the results of MCS for $10^{2}$ and $10^{3}$ samples are shown.

From Fig.8, it can be observed that the URP method with 2nd-order Taylor series approximation may under- 
(a)

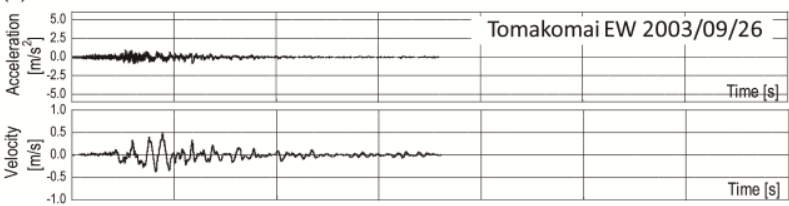

(b)

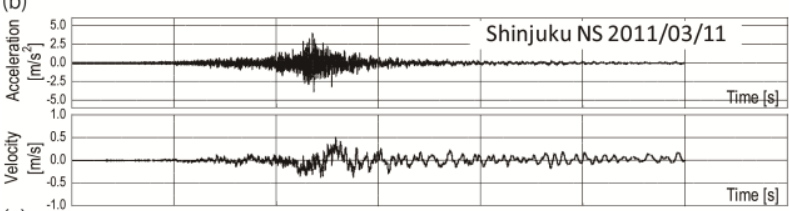

(c)

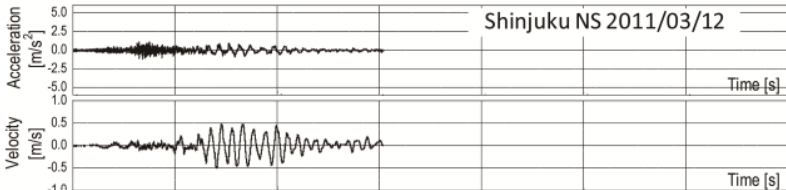

(d)

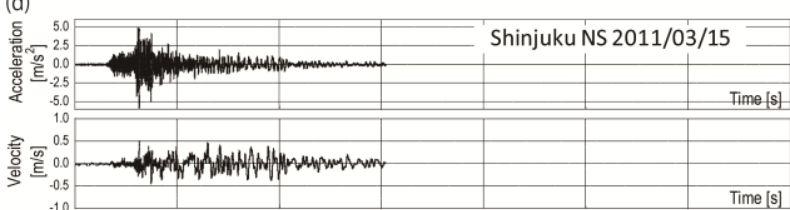

(e)

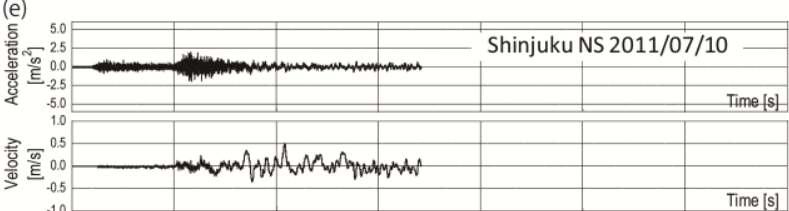

(f)

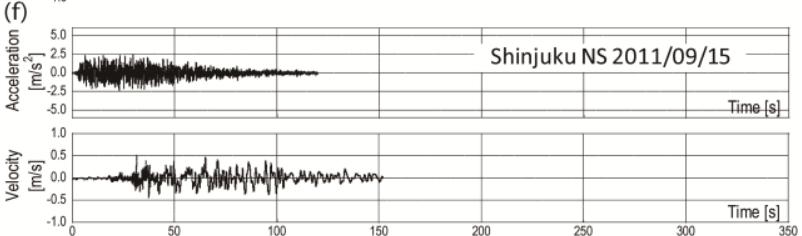

Fig.5 Recorded ground motions (Max.Vel. is $0.5 \mathrm{~m} / \mathrm{s}$ )

estimate the upper bound of the objective function predicted by MCS. This may result from the difficulty in the evaluation of the sensitivities for non-linear structural response, i.e. elastic-plastic behavior of the isolators. On the other hand, the result by the URP method with RSM coincides well with the upper bound of the MCS and this indicates the usefulness and reliability of the URP method with RSM. The upper bound of the maximum drift of base-isolation story is nearly $1.5[\mathrm{~m}]$ for the ground motion of Shinjuku NS $(2011 / 03 / 12)$. This may be due to the resonant phenomenon where the dominant frequency of the ground motion coincides with the natural frequency of the base-isolated building. However, it should be mentioned that Shinjuku NS (2011/03/12) is regarded as

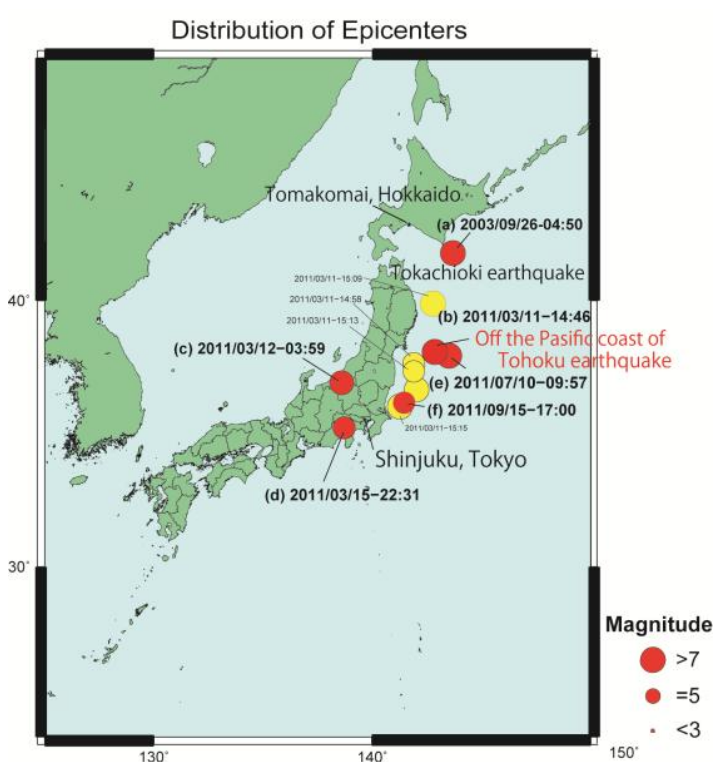

Fig.6 Distribution of epicenters [18]

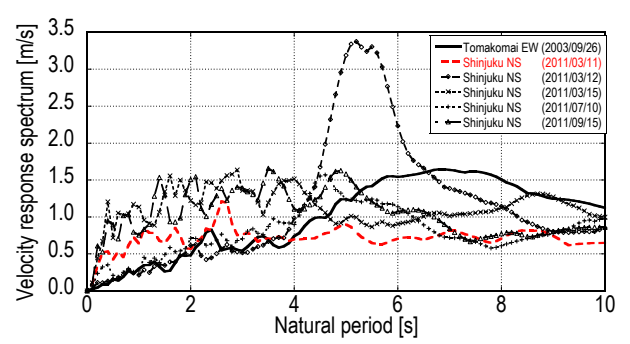

Fig.7 Velocity response spectrum $(h=0.05)$

one of the aftershock earthquake ground motions caused by the 2011 off the Pacific coast of Tohoku earthquake (2011/03/11) and the moment magnitude is much less than the main shock, i.e. the amplitude of Shinjuku NS (2011/03/12) has been increased dramatically through the normalization. On the other hand, for other ground motions, the maximum drift of the base-isolation story does not exhibit much difference in the nominal model. However it is also true that the level of variability of the responses due to the variability of structural parameters shows different properties depending on ground motions. This may result from the fact that the effect of variability of structural properties is correlated complicatedly with the frequency contents of ground motions. 
Fig.9 indicates the critical distribution of interval parameters for the URP method with 2nd-order Taylor series expansion, the URP method with RSM and the critical one derived by MCS. These distributions are obtained for 6 ground motions. The abscissa indicates the ratio of variability of interval parameters to the nominal value ( -1 means the lower bound of the interval parameter and 1 indicates the upper bound of the interval parameter). On the other hand, the ordinate presents the interval parameter number defined in Eq.(12). It can be observed that the upper bound of the objective function derived by the URP method with RSM almost coincides with that of MCS for several ground motions. By comparing the critical combinations of the interval parameters of Figs.9 (b) and (c) in the records of Tomakomai EW and Shinjuku NS (2011/03/12), the critical variations of the interval parameters of the isolator's property (i.e. the interval variable number $i=1,2,3,4)$ are given by the same combinations. This fact may lead to the acceptable coincidence between the proposed method and the critical solution derived by MCS. It can also be stated from Fig.9 that the upper bound of the objective function derived by the URP method with 2nd-order Taylor series approximation has the same value as that derived by MCS in the records of Shinjuku NS (2011/09/15). However, by comparing the critical combination of the interval parameters of the isolator's property in the same way, no coincidence among the isolator's property can be observed except the damping coefficient of the damper at the base-isolation story. As for the variation of structural parameters in the super structure, it may be difficult to find certain tendencies of the critical variation. It is well known [12] that the parameters in super structures do not influence much the drift in the base-isolation story.

Fig.10 shows the comparison of the hysteretic loop and time history of drift in the base-isolation story for models with nominal parameters and critical parameters under Shinjuku NS (2011/03/12). As seen in Fig.9, not only the drift of base-isolation story but also the restoring force of the isolator is varied from the nominal structural response.

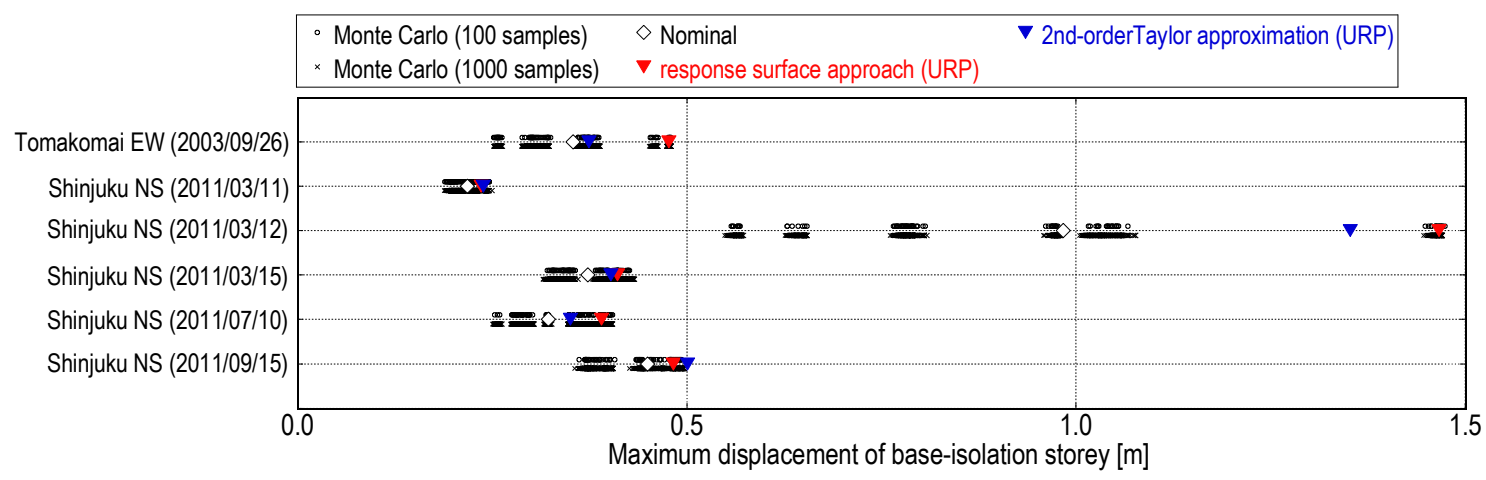

Fig.8 Comparison of maximum interstory drift of base-isolation story derived by URP method with MCS
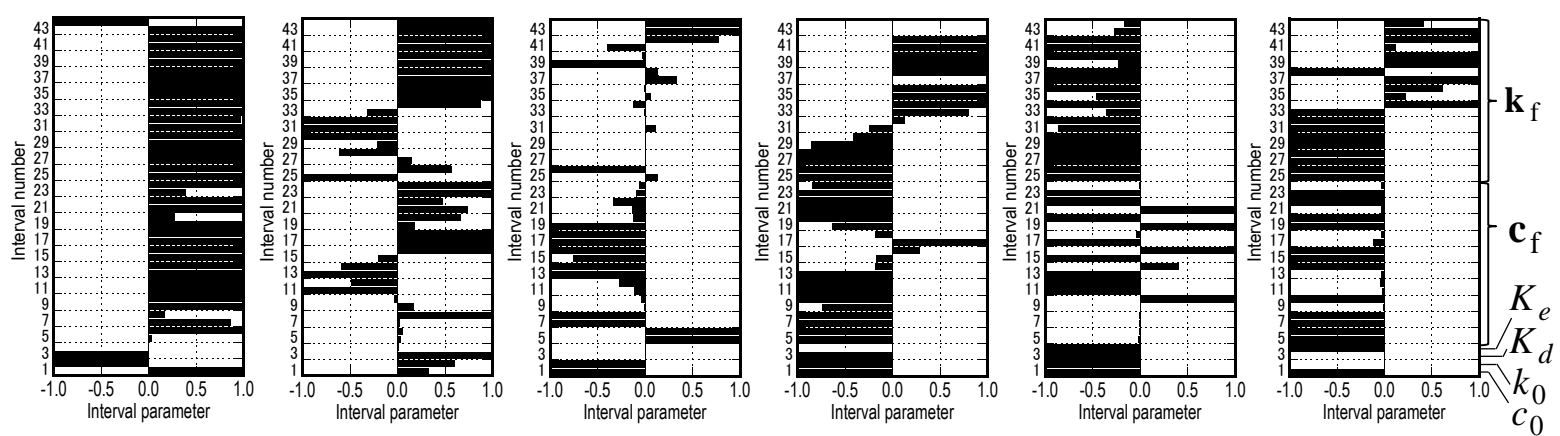

(a) URP method with 2nd-order Taylor series approximation

Fig.9 Critical distribution of uncertain parameters for drift of base-isolation story 

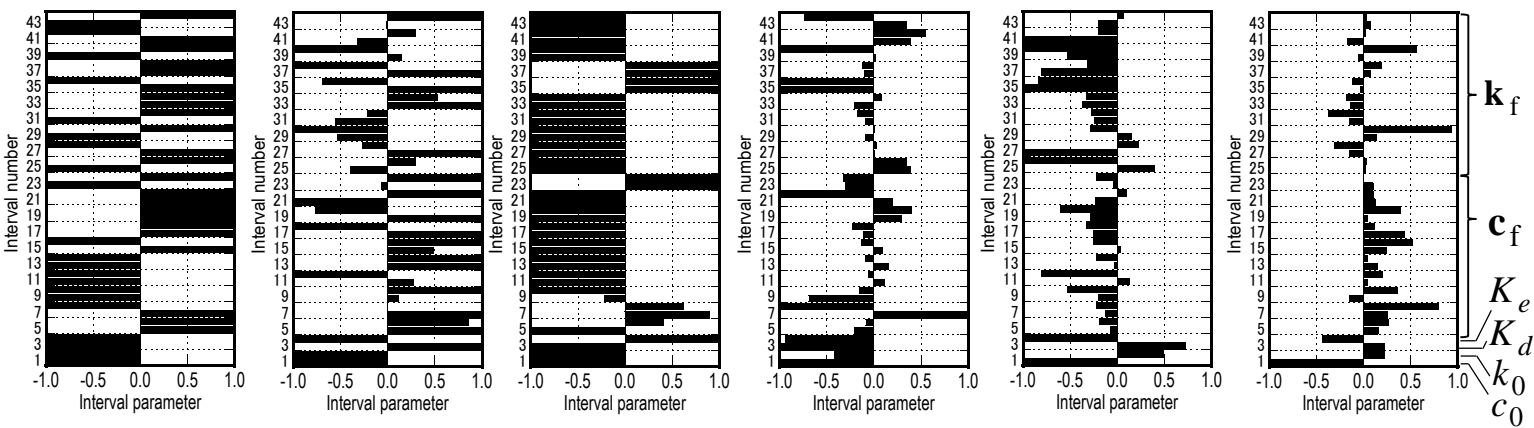

(b) URP method with response surface approach

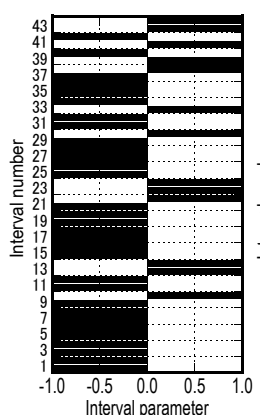

Tomakomai $(2003 / 09 / 26)$

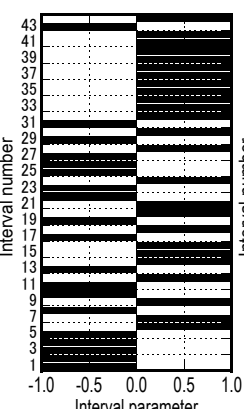

Shinjuku $(2011 / 03 / 11)$

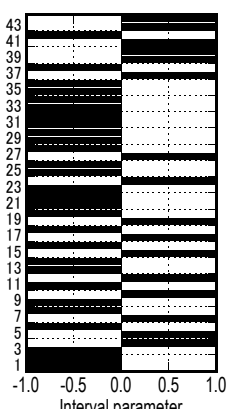

Shinjuku (2011/03/12)

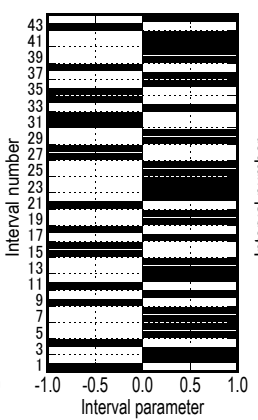

Shinjuku (2011/03/15)

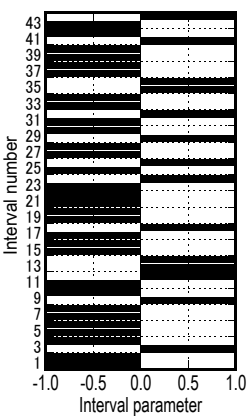

Shinjuku $(2011 / 07 / 10)$

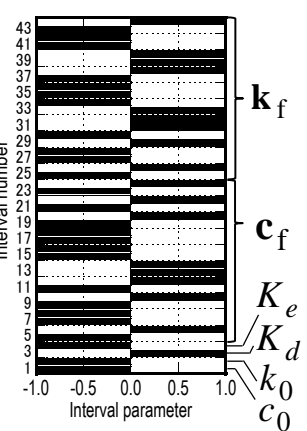

Shinjuku $(2011 / 09 / 15)$

(c) Monte Carlo method

Fig.9 Critical distribution of uncertain parameters for drift of base-isolation story (continued)

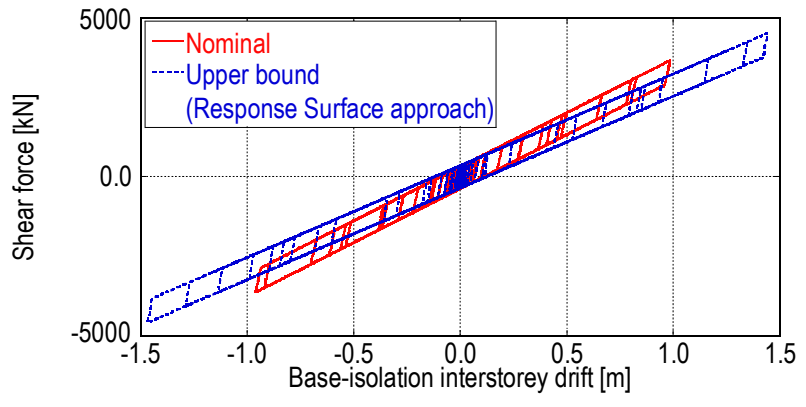

(a) Hysteresis loop

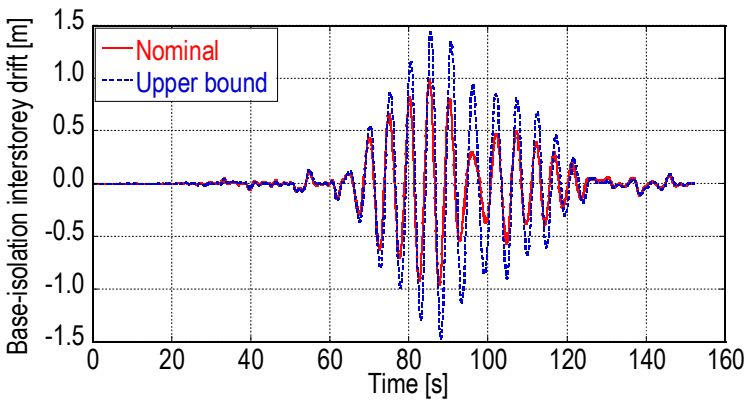

(b) Time history of drift in base-isolation story

Fig.10 Comparison of hysteretic loop and time history of drift in base-isolation story for models with nominal parameters and critical parameters under Shinjuku NS (2011/03/12).

4.5 Interval analysis for top-story maximum acceleration. Fig.11 presents the results, i.e. the upper bound of the maximum top-story floor acceleration, of interval analysis by various methods (URP methods with 2nd-order Taylor series approximation / with RSM and the Monte Carlo simulation). It can be observed from Fig.11 that the level of variability of the maximum value into the increasing side derived by the URP method with RSM is larger than that with 2 nd-order Taylor series approximation. This may result from the difficulty in the sensitivity analysis for elastic-plastic characteristics. Furthermore, it can be seen that the upper bounds of the objective function derived by the proposed method are larger than the results of MCS. As seen later in Fig.12, only the end points combination is not sufficient in MCS for evaluating the upper bound of the acceleration at the top story. On the other hand, the URP method with RSM provides a rather reliable evaluation. This indicates the usefulness and reliability of the URP method with RSM.

Fig.12 indicates the critical distribution of interval 
parameters for the URP methods with 2nd-order Taylor series approximation / with RSM and the critical one derived from MCS. From the investigation in Fig.12, it may be said that the URP method is effective. This is because the possibility is taken into account of occurrence of the extreme value of the objective function in an inner feasible region. Since the upper bounds of the objective function derived by each method are different, it is natural that the critical distribution of interval parameters may not coincide.

While, in usual structural design of base-isolated buildings, the variability of base-isolation systems is often taken into account, the variability of super-building parameters is not considered. As for the horizontal displacement of the base-isolation story, the variability of the base-isolation systems is essential. On the other hand, for the interstory drifts in super-buildings and top-story acceleration, the variability of super-building parameters is also significant. Therefore a detailed examination should be made carefully.

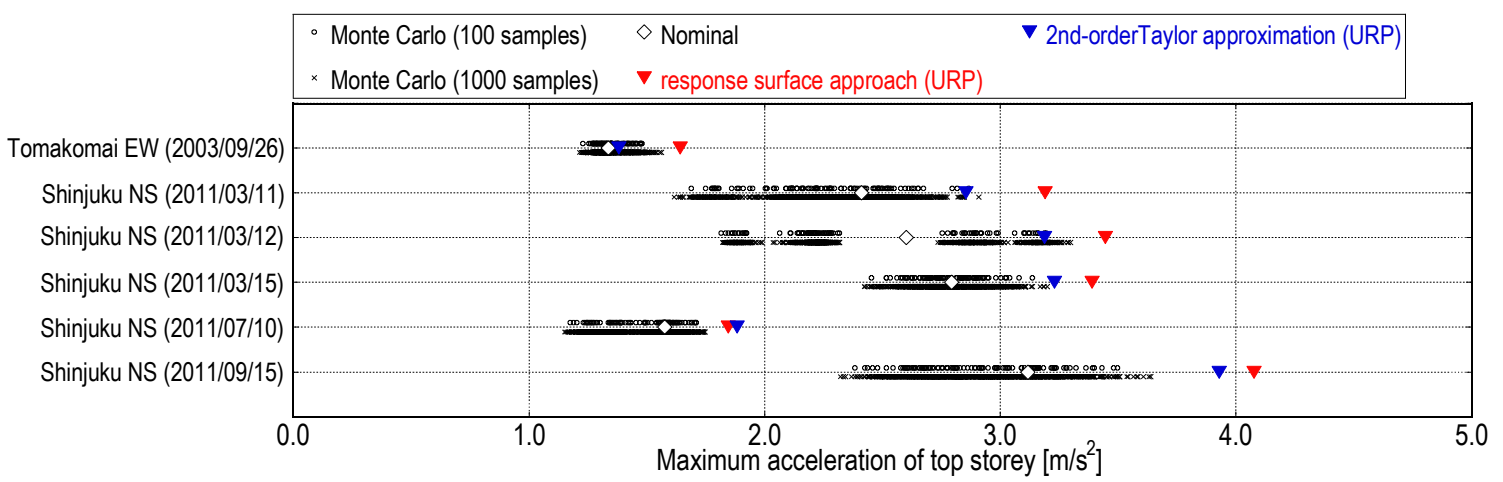

Fig.11 Comparison of maximum top-story acceleration derived by URP method with MCS
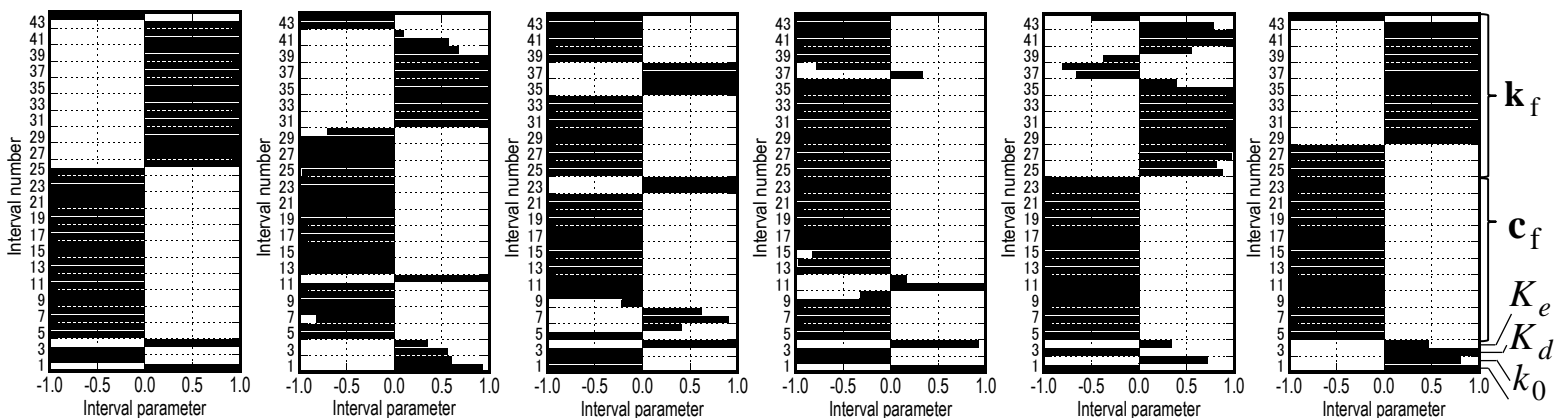

(a) URP method with 2nd-order Taylor series approximation
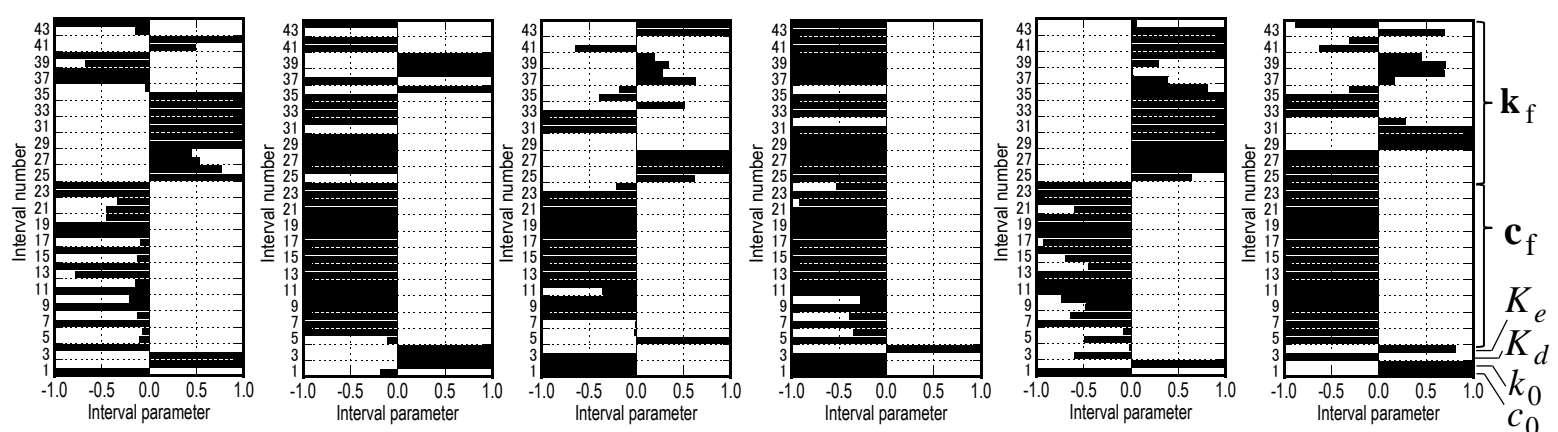

(b) URP method with response surface approach

Fig.12 Critical distribution of uncertain parameters for acceleration of top story 

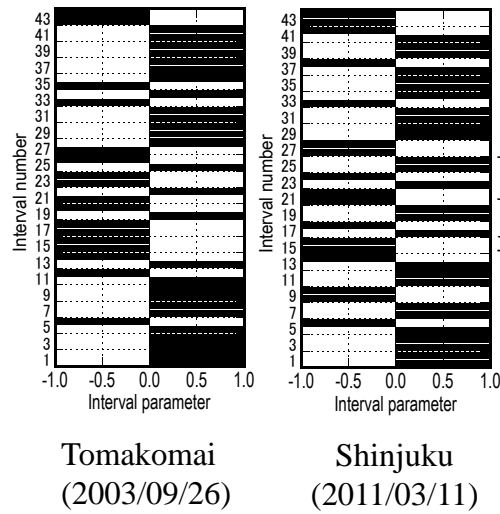
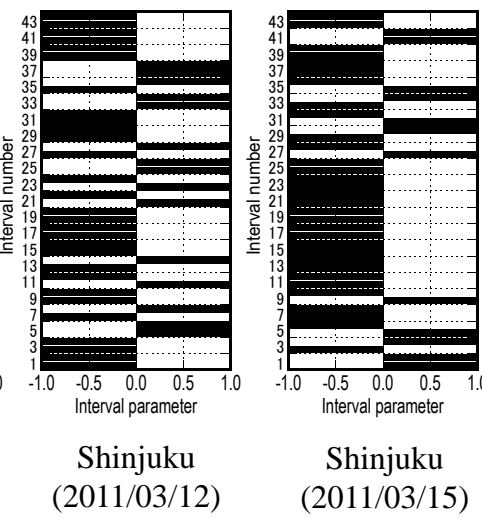
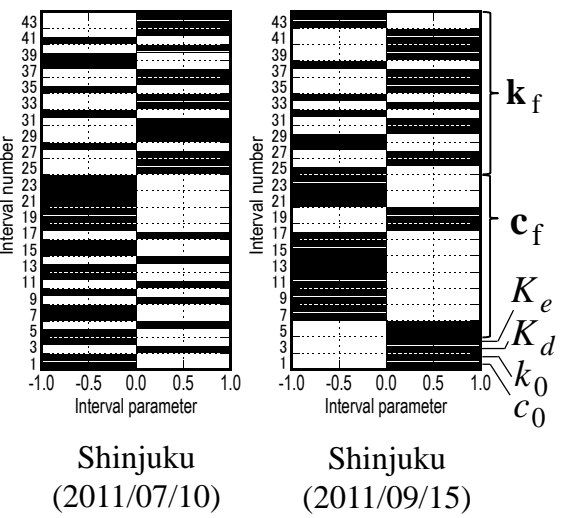

Shinjuku

(2011/09/15)

(c) Monte Carlo method

Fig.12 Critical distribution of uncertain parameters for acceleration of top story (continued)

\section{Conclusions}

An efficient interval analysis methodology for robustness evaluation of a base-isolated building has been presented to find the upper bound of the dynamic response under several long-period ground motions by using the URP (Updated Reference-Point) method with second-order Taylor series expansion and a response surface method. The objective function is defined by the maximum drift of the base-isolation story or the maximum acceleration at the top story. Since the critical combination of interval parameters in a feasible domain, not only on the bounds but also in an inner domain of interval parameters, has been derived explicitly, the upper bound of the objective function can be obtained straightforwardly for the predicted structural parameter set.

Since several long-period ground motions have been used as input motions and elastic-plastic responses are considered, numerical differentiation has to be used in the URP method for calculating sensitivities. It has been shown that, by using these sensitivities, the objective function can also be approximated by second-order Taylor series expansion including full elements.

When numerical differentiation has some difficulties, a response surface method provides a powerful means. It has been shown that, in the response surface method combined with the URP method, the variation of the objective function can be evaluated successfully from the responses at the three points.

Numerical examples of the robustness evaluation have been presented for a 20 -story base-isolated shear building with elastic-plastic isolators by applying the URP method and the Monte Carlo method.

Although the critical distribution of interval parameters exhibits a unstable tendency, the upper bounds of specific objective functions (drift of base-isolation story or top-story acceleration) by three methods (URP with 2nd-order Taylor series expansion, URP with a response surface approximation and the Monte Carlo simulation) show good correspondence. It has been shown that the URP method combined with a response surface approximation possesses an excellent accuracy and reliability.

\section{Acknowledgement}

Part of the present work is supported by the Grant-in-Aid for Scientific Research of Japan Society for the Promotion of Science (No.20656086, 21360267). This support is greatly appreciated.

\section{Notes and References}

[1] Architectural Institute of Japan (2001). Recommendation for Design of Base Isolated buildings (in Japanese).

[2] Japan Society of Seismic Isolation (JSSI) (2010). Base-isolated structures - from fundamentals to design and practice (in Japanese).

[3] Naeim, F. and Kelly, JM. (1999). Design of Seismic Isolated Structures. Wiley: New York.

[4] Higashino, M. and Wada, A. (2009). A study on seismic isolation system with robustness for increasing ground motion level, J. Structural and Construction Engineering, No.641, pp1233-1240 (in Japanese).

[5] Zhao, Y., Ono, T. and Suzuki, M. (1997). Dynamic structural reliability evaluation considering parameter uncertainties, J. Structural and Construction Engineering, No.497, pp33-38 (in Japanese). 
[6] Takewaki, I. (2008). Robustness of base-isolated high-rise buildings under code-specified ground motions, Struct. Design Tall Spec. Build., 17, pp257-271.

[7] Ben-Haim, Y. and Elishakoff, I. (1990). Convex models of uncertainty in applied mechanics. Amsterdam: Elsevier.

[8] Kanno, Y. and Takewaki, I. (2005). Approximation algorithm for robustness functions of trusses with uncertain stiffness under uncertain forces, $J$. Structural and Construction Engineering, No.591, pp53-60 (in Japanese).

[9] Kanno, Y. and Takewaki, I. (2006). Robustness analysis of trusses with separable load and structural uncertainties, Int. J. Solids and Structures, Vol.43, No.9 pp.2646-2669.

[10] Ben-Haim Y. (2001). Information-gap decision theory: decisions under severe uncertainty. Academic Press, San Diego.

[11] Takewaki, I. and Ben-Haim, Y. (2005). Info-gap robust design with load and model uncertainties, Special Issue: Uncertainty in Structural Dynamics, $J$. Sound \& Vibration, 288(3), pp551-570.

[12] Fujita, K. and Takewaki, I. (2011). Earthquake response bound analysis of uncertain base-isolated buildings for robustness evaluation, J. Structural and
Construction Engineering, No.666, pp1453-1460 (in Japanese).

[13] Fujita, K. and Takewaki, I. (2011). An efficient methodology for robustness evaluation by advanced interval analysis using updated second-order Taylor series expansion, Eng. Struct, Vol.33, No.12, pp3299-3310.

[14] Rao, S. and Berke, L. (1997). Analysis of uncertain structural systems using interval analysis, AIAA Journal, 34 (4), pp727-735.

[15] Qiu, Z. and Elishakoff, I. (1998). Antioptimization of structures with large uncertain-but-nonrandom parameters via interval analysis, Comp. Meth. Appl. Mech. Engrg., 152, pp361-372.

[16] Qiu, Z., Chen, S., Song, D. (1996). The displacement bound estimation for structures with an interval description of uncertain parameters, $C$. Numer. Meth Engng., 12, pp1-11.

[17] Chen, S., Ma, L., Meng, G. and Guo, R. (2009). An efficient method for evaluating the natural frequency of structures with uncertain-but-bounded parameters, Comp. Struct., 87, pp582-590.

[18] NIED (2012). http://www.k-net.bosai.go.jp/k-net/ (accessed on 5, March, 2012), Fig.5 has been edited from the epicenter distribution map of each month. 